\title{
Hybrid enriched bidiagonalization for discrete ill-posed problems
}

\author{
Hansen, Per Christian; Dong, Yiqiu; Abe, Kuniyoshi
}

Published in:

Numerical Linear Algebra with Applications

Link to article, DOI:

$10.1002 /$ nla.2230

Publication date:

2019

Document Version

Peer reviewed version

Link back to DTU Orbit

Citation (APA):

Hansen, P. C., Dong, Y., \& Abe, K. (2019). Hybrid enriched bidiagonalization for discrete ill-posed problems. Numerical Linear Algebra with Applications, 26(3), [e2230]. https://doi.org/10.1002/nla.2230

\section{General rights}

Copyright and moral rights for the publications made accessible in the public portal are retained by the authors and/or other copyright owners and it is a condition of accessing publications that users recognise and abide by the legal requirements associated with these rights.

- Users may download and print one copy of any publication from the public portal for the purpose of private study or research.

- You may not further distribute the material or use it for any profit-making activity or commercial gain

- You may freely distribute the URL identifying the publication in the public portal

If you believe that this document breaches copyright please contact us providing details, and we will remove access to the work immediately and investigate your claim. 
DOI: $\mathrm{xxx} / \mathrm{xxxx}$

\title{
RESEARCH ARTICLE
}

\section{Hybrid enriched bidiagonalization for discrete ill-posed problems}

\author{
Per Christian Hansen*1 | Yiqiu Dong ${ }^{1}$ | Kuniyoshi Abe $^{2}$
}

${ }^{1}$ Department of Applied Mathematics and Computer Science, Technical University of Denmark, Kgs. Lyngby, Denmark

${ }^{2}$ Faculty of Economics and Information, Gifu Shotoku Gakuen University, Gifu, Japan

Correspondence

*Per Christian Hansen, Department of Applied Mathematics and Computer Science, Technical University of Denmark, Kgs. Lyngby, Denmark. Email: pcha@dtu.dk

\section{Funding information}

This work was supported by the European Research Council under Grant No. 291405 (HD-Tomo) and Grant No. 11701388 from the National Natural Science Foundation of China.

\begin{abstract}
The regularizing properties of the Golub-Kahan bidiagonalization algorithm are powerful when the associated Krylov subspace captures the dominating components of the solution. In some applications the regularized solution can be further improved by enrichment, i.e., by augmenting the Krylov subspace with a low-dimensional subspace that represents specific prior information. Inspired by earlier work on GMRES we demonstrate how to carry these ideas over to the bidiagonalization algorithm, and we describe how to incorporate Tikhonov regularization. This leads to a hybrid iterative method where the choice of regularization parameter in each iteration also provides a stopping rule.
\end{abstract}

\section{KEYWORDS:}

Krylov subspace methods, regularizing iterations, enriched subspaces, hybrid iterative methods

\section{1 | INTRODUCTION}

We are concerned with iterative Krylov subspace methods for solving large ill-conditioned systems on linear equations, arising from discretization of inverse problems, of the form

$$
\min _{x}\|A x-b\|_{2}^{2}, \quad A \in \mathbb{R}^{m \times n} .
$$

Such problems arise in many areas of science and engineering ${ }^{1}$ and it is important to have access to efficient algorithms that can handle large-scale inverse problems. To compute a stable solution to such problems, one must incorporate prior information about the desired solution. One often chooses a variational formulation known as Tikhonov regularization,

$$
\min _{x}\left\{\|A x-b\|_{2}^{2}+\lambda^{2} \mathcal{R}(x)\right\} .
$$

Here $\mathcal{R}(x)$ is a regularization or smoothness term that penalizes unwanted features in the solution, and $\lambda$ is a user-chosen regularization parameter ${ }^{1]}$.

Instead of enforcing smoothness conditions on the solution, one may have prior information that can be specified in the form of a low-dimensional subspace in which the solution must lie ${ }^{1}$. This leads to a projection formulation of the form

$$
\min _{x}\|A x-b\|_{2}^{2} \quad \text { s.t. } \quad x \in \mathcal{S}_{k},
$$

where the signal subspace $S_{k}$ is a linear subspace of dimension $k$. If the basis of $S_{k}=\operatorname{span}\left\{v_{1}, v_{2}, \ldots, v_{k}\right\}$ is chosen such that it captures the main features in the solution, then this approach can be very useful. The latter approach is particularly attractive for large-scale problems, where the signal subspace can take the form of a Krylov subspace, such as: 


$$
\begin{aligned}
& \mathcal{K}_{k}=\operatorname{span}\left\{A^{T} b, A^{T} A A^{T} b,\left(A^{T} A\right)^{2} A^{T} b, \ldots,\left(A^{T} A\right)^{k-1} A^{T} b\right\} \quad \text { for the CGLS and LSQR algorithms }{ }^{102}, \\
& \overline{\mathcal{K}}_{k}=\operatorname{span}\left\{b, A b, A^{2} b, \ldots, A^{k-1} b\right\} \quad \text { for the GMRES and MINRES algorithms }{ }^{3} \cdot 4 \text {, } \\
& \overrightarrow{\mathcal{K}}_{k}=\operatorname{span}\left\{A b, A^{2} b, A^{3} b, \ldots, A^{k} b\right\} \quad \text { for the RRGMRES and MR-II algorithms }{ }^{5}[6,
\end{aligned}
$$

where $k$ is the number of iterations; such methods are sometimes referred to as regularizing iterations. Depending on the application, one or more of these subspaces may be well suited to compute a good regularized solution, i.e., a good approximation that is only little sensitive to perturbations of the data ${ }^{7}$. Moreover, it is possible to combine the projection formulation with Tikhonov regularization; this leads to so-called hybrid iterative methods ${ }^{1 / 8}$ where Tikhonov regularization is applied in each iteration of the algorithm to the projected problem, i.e., the problem of small dimensions in the relevant Krylov subspace.

We can further improve the regularized solution by incorporating additional specific prior information. In this work we assume that the solution has a significant component in a given subspace $\mathcal{W}_{p}$ of dimension $p \ll k$ (e.g., chosen to represent known features in the solution). In connection with the above Krylov subspace methods, it was proposed ${ }^{9}[10$ to decompose the solution into a component in $\mathcal{W}_{p}$ and another component in the orthogonal complement $\mathcal{W}_{p}^{\perp}$, which leads to the idea of enriched (or augmented) Krylov subspace methods. See also $\frac{11}{11}$ for an adaptive approach.

Recently we presented an algorithm $\mathrm{R}^{3}$ GMRES ${ }^{[12}$ based on the range-restricted GMRES (RRGMRES) method ${ }^{[5]}$ and the corresponding Krylov subspace $\overrightarrow{\mathcal{K}}_{k}$. In the present work we consider a similar approach based on the LSQR method and the underlying Golub-Kahan bidiagonalization algorithm 13 , and the corresponding Krylov subspace $\mathcal{K}_{k}$. Specifically, we compute regularized solutions in a signal subspace $S_{p, k}$ that is the direct sum of the two subspaces $\mathcal{W}_{p}$ and $\mathcal{K}_{k}$,

$$
S_{p, k}=\mathcal{W}_{p}+\mathcal{K}_{k} \equiv\left\{y+z \mid y \in \mathcal{W}_{p} \wedge z \in \mathcal{K}_{k}\right\},
$$

which itself is a linear subspace.

An efficient and stable algorithm ENRICHED CGNR for this problem, based on the CGLS algorithm, was published in ${ }^{10}$. Here we present an alternative algorithm that takes its basis in the LSQR algorithm and the underlying bidiagonalization process. Due to this formulation, our algorithm lends itself easily to extensions to hybrid iterative algorithms where an additional Tikhonv regularization terms is incorporated, and where it is possible to efficiently compute the corresponding regularization parameter in each iteration. The latter also provides a stopping rule for the iterations, when the regularization parameter stabilizes.

Our paper is organized as follows. We first present the basic enriched bidiagonalization algorithm and discuss how to implement it. Then we introduce Tikhonov regularization which leads to the hybrid version of the algorithm, and again we discuss implementation aspects. We conclude with a few illustrative numerical examples. Throughout, $I_{q}$ denotes an identity matrix of order $q$.

\section{2 | FORMULATION OF THE BASIC ALGORITHM}

We want to solve the problem

$$
\min _{x}\|A x-b\|_{2}^{2} \quad \text { s.t. } \quad x \in \mathcal{W}_{p}+\mathcal{K}_{k} .
$$

In principle we could use, say, a Hessenberg decomposition

$$
A\left[W_{p}, A^{T} b, A^{T} A A^{T} b, \ldots,\left(A^{T} A\right)^{k-1} A^{T} b\right]=V_{p+k+1} H_{p+k}
$$

and compute the solution as

$$
\begin{aligned}
& x^{(k)}=\left[W_{p}, A^{T} b, A^{T} A A^{T} b, \ldots,\left(A^{T} A\right)^{k-1} A^{T} b\right] y^{(k)}, \\
& y^{(k)}=\operatorname{argmin}_{y}\left\|H_{p+1} y-V_{p+k+1}^{T} b\right\|_{2}^{2} .
\end{aligned}
$$

But we prefer to use a stable and efficient "standard" algorithm. Following the ideas behind the $\mathrm{R}^{3}$ GMRES algorithm ${ }^{[12}$ for square matrices, we use the bidiagonalization algorithm to compute an orthonormal basis of $\mathcal{K}_{k}$, and augment it by $\mathcal{W}_{p}$ in each iteration of the algorithm. This may seem cumbersome - but the overhead is, in fact, favorably small.

At iteration $k$ we have the decomposition

$$
A\left[V_{k}, W_{p}\right]=\left[U_{k+1}, \widetilde{U}_{k}\right]\left[\begin{array}{cc}
B_{k} & G_{k} \\
0 & F_{k}
\end{array}\right],
$$


where $V_{k}, U_{k+1}$ and $B_{k}$ are associated with the classical bidiagonalization algorithm. The remaining matrices are associated with the enrichment/augmentation. Specifically:

- $A V_{k}=U_{k+1} B_{k}$ is obtained after $k$ iterations of the bidiagonalization process.

- $V_{k} \in \mathbb{R}^{n \times k}$ has orthonormal columns that $\operatorname{span} \mathcal{K}_{k}$.

- $U_{k+1} \in \mathbb{R}^{m \times(k+1)}$ has orthonormal columns, and $u_{1}=b /\|b\|_{2}$.

- $B_{k} \in \mathbb{R}^{(k+1) \times k}$ is a lower bidiagonal matrix.

- $\widetilde{U}_{k} \in \mathbb{R}^{m \times p}$ satisfies range $\left(A W_{p}\right)=\operatorname{range}\left(U_{k+1} G_{k}+\widetilde{U}_{k} F_{k}\right)$ and $\widetilde{U}_{k}^{T} U_{k+1}=0$.

- $F_{k} \in \mathbb{R}^{p \times p}$ and changes in every iteration.

- $G_{k}$ is $(k+1) \times p$ and is updated associated with $U_{k+1}$.

The columns of $\left[V_{k}, W_{p}\right]$ form a basis for $S_{p, k}$. The matrices $G_{k} \in \mathbb{R}^{(k+1) \times p}$ and $F_{k} \in \mathbb{R}^{p \times p}$ are composed of the coefficients of $A W_{p}$ with respect to basis of $\operatorname{range}\left(U_{k+1}\right)$ and $\operatorname{range}\left(\widetilde{U}_{k}\right)$, respectively:

$$
G_{k}=U_{k+1}^{T} A W_{p}, \quad F_{k}=\widetilde{U}_{k}^{T} A W_{p} .
$$

Then the iterate $x^{(k)} \in S_{p, k}$ is given by $x^{(k)}=\left[V_{k}, W_{p}\right] y^{(k)}$, where $y^{(k)}$ solves a least squares problem:

$$
y^{(k)}=\operatorname{argmin}_{y}\left\|\left[\begin{array}{cc}
B_{k} & G_{k} \\
0 & F_{k}
\end{array}\right] y-\left[\begin{array}{c}
U_{k+1}^{T} \\
\widetilde{U}_{k}^{T}
\end{array}\right] b\right\|_{2}^{2} .
$$

The above derivation leads to the following generic formulation:

\section{BASIC ENRICHED BIDIAGONALIZATION}

1. Set $U_{1}=b /\|b\|_{2}, V_{0}=[], B_{0}=[], G_{0}=U_{1}^{T} A W_{p}, k=1$.

2. Use the bidiagonalization process to obtain $v_{k}$ and $u_{k+1}$ such that $A V_{k}=U_{k+1} B_{k}$, where

$$
V_{k}=\left[V_{k-1}, v_{k}\right], U_{k+1}=\left[U_{k}, u_{k+1}\right], B_{k}=\left[\begin{array}{cc} 
& 0 \\
B_{k-1} & \times \\
0 & \times
\end{array}\right] \text {. }
$$

3. Compute $G_{k}=\left[\begin{array}{c}G_{k-1} \\ u_{k+1}^{T} A W_{p}\end{array}\right] \in \mathbb{R}^{(k+1) \times p}$.

4. Orthonormalize $A W_{p}$ with respect to $U_{k+1}$ to obtain $\widetilde{U}_{k} \in \mathbb{R}^{m \times p}$.

5. Compute $F_{k}=\widetilde{U}_{k}^{T} A W_{p} \in \mathbb{R}^{p \times p}$.

6. Solve $\min _{y}\left\|\left[\begin{array}{cc}B_{k} & G_{k} \\ 0 & F_{k}\end{array}\right] y-\left[\begin{array}{c}U_{k+1}^{T} \\ \widetilde{U}_{k}^{T}\end{array}\right] b\right\|_{2}^{2}$ to obtain $y^{(k)}$.

7. Then $x^{(k)}=\left[V_{k}, W_{p}\right] y^{(k)}$.

8. Stop, or set $k:=k+1$ and return to Step 2 .

We note that we need to recompute the skinny $m \times p$ matrix $\widetilde{U}_{k}$ and the small $p \times p$ matrix $F_{k}$ in each iteration, but the dimension $p$ of the augmentation subspace is small so this overhead is negligible.

For efficiency reasons when solving the least squares problem in Step 6, in each iteration we update the orthogonal factorization:

$$
\left[\begin{array}{cc}
B_{k} & G_{k} \\
0 & F_{k}
\end{array}\right]=Q\left[\begin{array}{cc}
T_{k}^{(11)} & T_{k}^{(12)} \\
0 & T_{k}^{(22)} \\
0 & 0
\end{array}\right]
$$



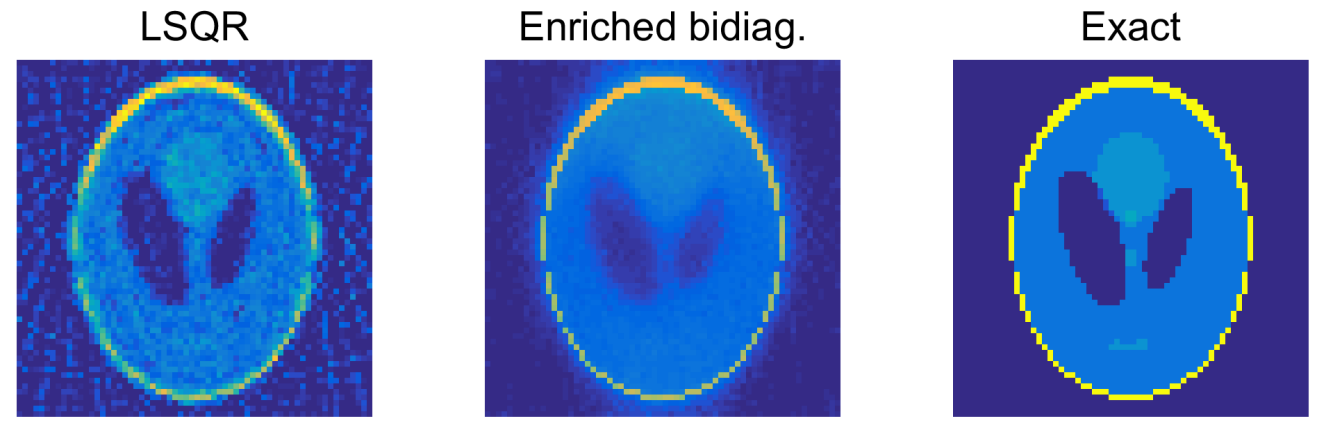

FIGURE 1 The Shepp-Logan head phantom and two reconstructions in which negative pixels are truncated to 0. The LSQR reconstruction shows the usual line artifacts associated with few-projection CT problems. On the other hand, the reconstruction computed with the enriched bidiagonalization method (as well as enriched CGNR) has much fewer artifacts.

where $T_{k}^{(11)} \in \mathbb{R}^{k \times k}$ and $T_{k}^{(22)} \in \mathbb{R}^{p \times p}$ are upper triangular, and $Q \in \mathbb{R}^{(k+p+1) \times(k+p+1)}$ is orthogonal. We update $T_{k}^{(11)}$ via Givens rotations that are also applied to $G_{k}$ and $U_{k+1}^{T} b$. The matrix $\widetilde{U}_{k}$ is already orthogonal to $U_{k}$, hence (in principle) we can perform the update $\widetilde{U}_{k+1}=\left(I_{m}-u_{k+1} u_{k+1}^{T}\right) \widetilde{U}_{k}$. For numerical stability, we must reorthogonalize the columns of $V_{k}, U_{k+1}$, and $\widetilde{U}_{k}$. This is an acceptable approached used in many similar algorithms, such as the algorithm HyBR ${ }^{14}$.

We emphasize that the iterates produced by our algorithm are mathematically identical to those produced by the enriched CGNR method 10 .

To illustrate the power of incorporating additional information via the augmentation subspace $\mathcal{W}_{p}$, we consider a parallelbeam X-ray CT problem generated by means of the function paralleltomo from the software package AIR ToOLS II ${ }^{[15}$. In this example, the $64 \times 64$ test image is the Shepp-Logan head phantom and our prior information is that the image has a particular oval structure that represents the skull. Hence we can choose binary basis vectors of $\mathcal{W}_{p}$ that represent this structure.

We use 22 projections of 91 pixels, so the matrix is $2002 \times 4096$ and the problem is underdetermined. We add $1 \%$ Gaussian noise to the data. We then solve the CT reconstruction problem by means of LSQR and the basic enriched bidiagonalization algorithm. The results are shown in Figure 1 where we see that incorporation of the specific information about the skull gives a much improved reconstruction, compared to that from the LSQR algorithm.

\section{3 | FORMULATION OF THE HYBRID ALGORITHM}

For some inverse problems, the Krylov subspace or the enriched subspace may not provide enough regularization, and an additional regularization term $\lambda^{2} \mathcal{R}(x)$ is needed. Here we consider the case where $\mathcal{R}(x)=\|x\|_{2}^{2}$ and the corresponding problem

$$
\min _{x}\left\{\|A x-b\|_{2}^{2}+\lambda^{2}\|x\|_{2}^{2}\right\} \quad \text { s.t. } \quad x \in S_{p, k} .
$$

After reformulating [11] as

$$
\min _{x}\left\{\left\|\left[\begin{array}{c}
A \\
\lambda I
\end{array}\right] x-\left[\begin{array}{c}
b \\
0
\end{array}\right]\right\|_{2}^{2}\right\} \quad \text { s.t. } \quad x \in S_{p, k},
$$

both the enriched CGNR algorithm and our enriched bidiagonalization algorithm can handle this problem efficiently, by adding a Tikhonov regularization term to the projected problem in each iteration. The details on how such a term is added to the LSQR algorithm can be found in Section 6.4 and Fig. 6.10 in 1 .

A further advantage of adding a regularization term to the projected problem is that, in some case, we can choose a different regularization parameter $\lambda_{k}$ in each iteration. Moreover, since the projected problems have much smaller dimensions than the original problem (1) we are free to use a variety of parameter-choice rules, including those that require the computation of the SVD of the projected matrix. This is often referred to as a hybrid iterative method and it is the basis of the HyBR algorithm 14 that applies this idea to the LSQR algorithm, using weighted generalized cross validation to choose the regularization parameter. Similar hybrid iterative algorithms based on (flexible) GMRES are implemented in the IR TooLs package 6 . 
The way that Tikhonov regularization is introduced in the enriched CGNR algorithm $\frac{10}{10}$ does not allow the regularization parameter to change in each iteration. On the other hand, since our algorithm explicitly builds on the bidiagonalization that also underlies LSQR, we can indeed introduce an iteration-dependent regularization parameter $\lambda_{k}$. Specifically, we obtain the following algorithm where PCR denotes the chosen parameter-choice rule:

\section{HYBRID ENRICHED BIDIAGONALIZATION}

1. Set $U_{1}=b /\|b\|_{2}, V_{0}=[], B_{0}=[], G_{0}=U_{1}^{T} A W_{p}, k=1$.

2. Use the bidiagonalization process to obtain $v_{k}$ and $u_{k+1}$ such that $A V_{k}=U_{k+1} B_{k}$, where

$$
V_{k}=\left[V_{k-1}, v_{k}\right], U_{k+1}=\left[U_{k}, u_{k+1}\right], B_{k}=\left[\begin{array}{cc} 
& 0 \\
B_{k-1} & \times \\
0 & \times
\end{array}\right] \text {. }
$$

3. Compute $G_{k}=\left[\begin{array}{c}G_{k-1} \\ u_{k+1}^{T} A W_{p}\end{array}\right] \in \mathbb{R}^{(k+1) \times p}$.

4. Orthonormalize $A W_{p}$ with respect to $U_{k+1}$ to obtain $\widetilde{U}_{k} \in \mathbb{R}^{m \times p}$.

5. Compute $F_{k}=\widetilde{U}_{k}^{T} A W_{p} \in \mathbb{R}^{p \times p}$.

6. Use PCR to choose $\lambda_{k}$ and compute $y_{\lambda_{k}}^{(k)}$ by solving the regularized problem

$$
\min _{y}\left\|\left[\begin{array}{cc}
B_{k} & G_{k} \\
0 & F_{k} \\
\lambda_{k} V_{k} & \lambda_{k} W_{p}
\end{array}\right] y-\left[\begin{array}{c}
U_{k+1}^{T} \\
\widetilde{U}_{k}^{T} \\
0
\end{array}\right] b\right\|_{2}^{2}
$$

7. Then $x^{(k)}=\left[V_{k}, W_{p}\right] y_{\lambda_{k}}^{(k)}$.

8. Stop, or set $k:=k+1$ and return to Step 2 .

Following the HyBR algorithm ${ }^{14}$ as well as the hybrid iterative algorithms in IR ToOLS ${ }^{16}$ we stop the iterations when the regularization parameter $\lambda_{k}$ stabilizes.

To solve the regularized problem in Step 6 efficiently, we already described how to update a QR factorization of the top $2 \times 2$ block matrix. To treat the large bottom block $\lambda_{k}\left[V_{k}, W_{p}\right] \in \mathbb{R}^{n \times(k+p)}$ efficiently, we multiply from the left with the orthogonal matrix $\left[V_{k}, V_{o}\right]^{T}$, where the columns of $V_{o}$ are chosen to make the full matrix orthogonal. The 2-norm is unaffected by this transformation, and the bottom block then takes the form

$$
\left[V_{k}, V_{o}\right]^{T} \lambda_{k}\left[V_{k}, W_{p}\right]=\lambda_{k}\left[\begin{array}{cc}
I_{k} & V_{k}^{T} W_{p} \\
0 & V_{o}^{T} W_{p}
\end{array}\right], \quad V_{k}^{T} W_{p}=\left[\begin{array}{c}
V_{k-1}^{T} W_{p} \\
v_{k}^{T} W_{p}
\end{array}\right],
$$

where we used that $V_{k}=\left[V_{k-1}, v_{k}\right]$ and $W_{p}^{T} v_{k}$ is a vector of length $p$. Since the matrix $V_{o}$ is not explicitly available, we consider instead the Cholesky factorization of the symmetric and positive definite $p \times p$ matrix

$$
\left(V_{o}^{T} W_{p}\right)^{T} V_{o}^{T} W_{p}=W_{p}^{T} V_{o} V_{o}^{T} W_{p}=W_{p}^{T}\left(I_{n}-V_{k} V_{k}^{T}\right) W_{p}=R_{k}^{T} R_{k},
$$

where $R_{k} \in \mathbb{R}^{p \times p}$ is the Cholesky factor. It follows immediately that

$$
R_{k}^{T} R_{k}=W_{p}^{T}\left(I_{n}-V_{k-1} V_{k-1}^{T}\right) W_{p}-\left(W_{p}^{T} v_{k}\right)\left(W_{p}^{T} v_{k}\right)^{T}=R_{k-1}^{T} R_{k-1}-\left(W_{p}^{T} v_{k}\right)\left(W_{p}^{T} v_{k}\right)^{T} .
$$

Hence we can compute $R_{k}$ from $R_{k-1}$ using techniques that downdate a Cholesky factor due to a rank-one change $\frac{17}{17}$, and the bottom block is reduced to a small matrix of size $(k+p) \times(k+p)$.

\section{4 | NUMERICAL EXAMPLES}

To illustrate the performance of our hybrid enriched bidiagonalization (HEB) algorithm we use the following approach:

1. Generate a noise-free system: $A x_{\text {exact }}=b_{\text {exact }}$.

2. Add noise: $b=b_{\text {exact }}+e$ where $e$ is a random vector of Gaussian white noise scaled such that $\|e\|_{2} /\left\|b_{\text {exact }}\right\|_{2}=\eta$. 

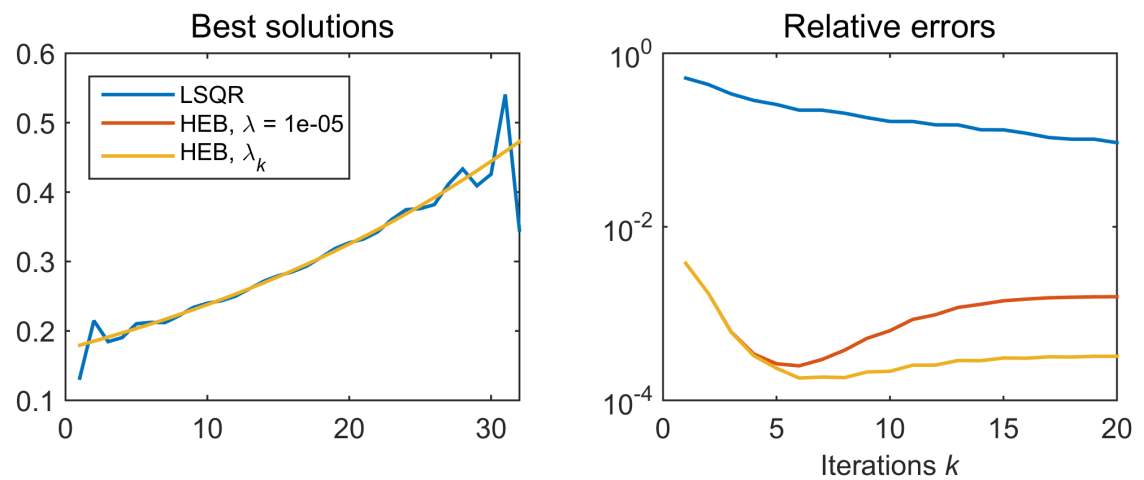

$\lambda_{k}$ chosen by GCV
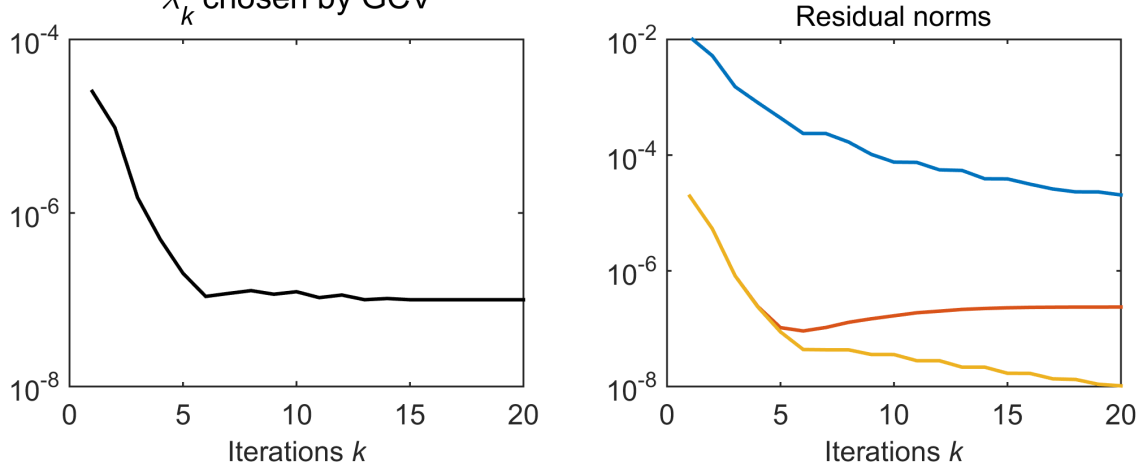

FIGURE 2 Results for the test problem deriv2 $(n, 2)$. LSQR is not able to produce a good solution with the Krylov subspace $\mathcal{K}_{k}$; HEB performs much better due to the subspace augmentation, and GCV successfully computes a very good regularization parameter $\lambda_{k}$ that stagnates (as it should) after 6 iterations. The exact solution is indistinguishable from the HEB solution.

3. Show the best solution within the iterations, the relative error $\left\|x_{\text {exact }}-x^{(k)}\right\|_{2} /\left\|x_{\text {exact }}\right\|_{2}$, and the residual norm $\left\|b-A x^{(k)}\right\|_{2}$. We compare the following three algorithms:

- LSQR is the implementation from REgULARIZATION TOOLS ${ }^{18}$.

- HEB with a fixed regularization parameter $\lambda$ chosen by the user.

- HEB with the regularization parameter $\lambda_{k}$ chosen by applying generalized cross validation (GCV) to the projected problem in each iteration.

\subsection{A large component in augmented subspace}

The first test problem is deriv2 $(\mathrm{n}, 2)$ from Regularization Tools ${ }^{18}$ with $n=32$ and relative noise level $\eta=10^{-6}$. The augmentation subspace is

$$
\mathcal{W}_{2}=\operatorname{span}\left\{w_{1}, w_{2}\right\}, \quad w_{1}=(1,1, \ldots, 1)^{T}, \quad w_{2}=(1,2, \ldots, n)^{T} .
$$

For this problem we have

$$
\left\|W_{2} W_{2}^{T} x_{\text {exact }}\right\|_{2} /\left\|x_{\text {exact }}\right\|_{2}=0.99, \quad\left\|\left(I_{n}-W_{2} W_{2}^{T}\right) x_{\text {exact }}\right\|_{2} /\left\|x_{\text {exact }}\right\|_{2}=0.035,
$$

and we only need to spend effort in capturing the small component of the solution in $\mathcal{W}_{2}^{\perp}$. But, as shown in the numerical results in Fig. 2, the augmentation approach is crucial for computing a feasible reconstruction without the artificial oscillations towards the ends produced by LSQR.

If we know a good value of the regularization parameter $\lambda$ then HEB can produce a good reconstruction. Without such prior knowledge, however, HEB produces less accurate reconstructions as demonstrated with the choice $\lambda=10^{-5}$ which is too large. 

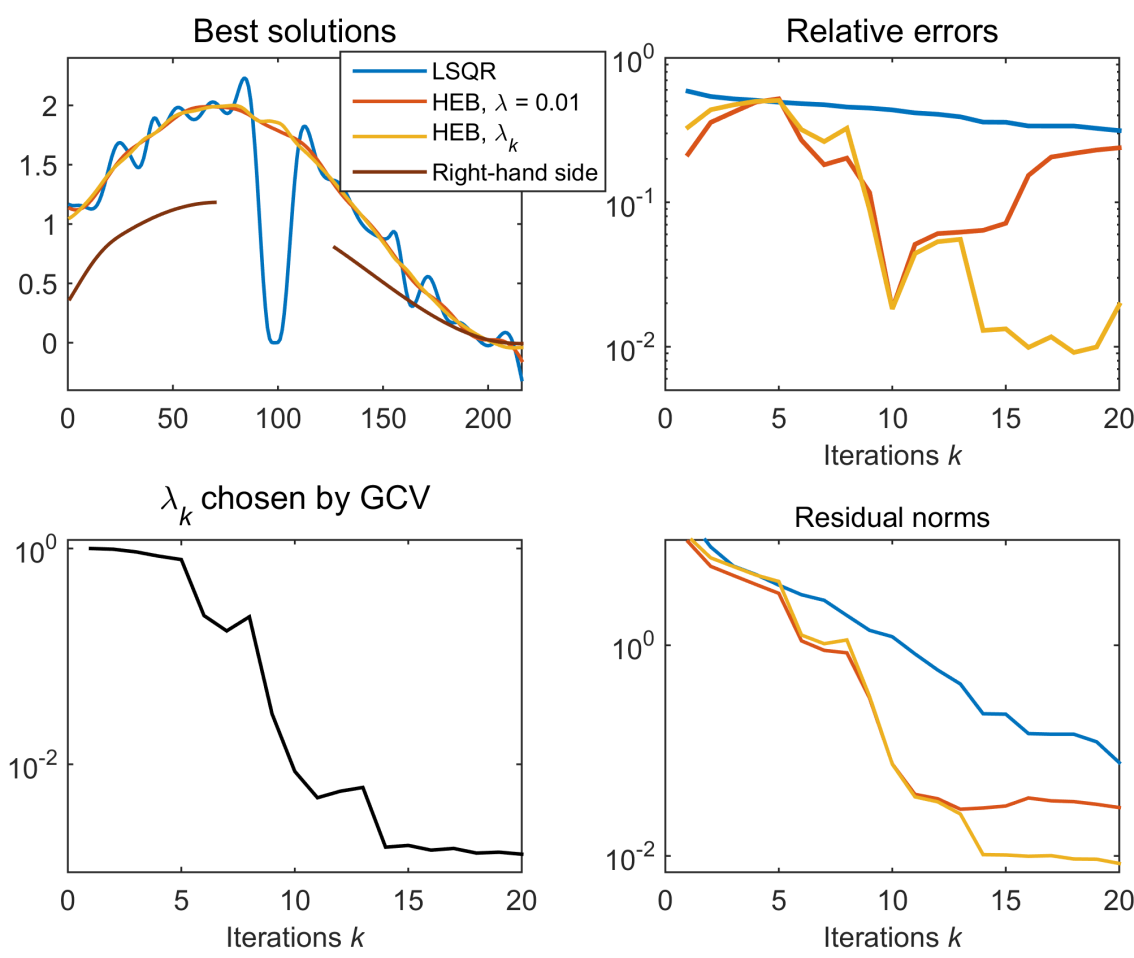

FIGURE 3 Numerical results for the test problem with combined deconvolution and inpainting. The top left plot shows the best reconstructions with the three methods, as well as the right-hand side with 56 "missing" values in the middle. The LSQR solution has many unwanted oscillations as well as a large error in the middle. The other two methods can produce good reconstructions, but only HEB with $\lambda_{k}$ chosen by GCV needs no prior knowledge about the noise level or the regularization parameter.

On the other hand, if we use GCV to adaptively choose $\lambda_{k}$ in each iteration then this parameter settles at approximately $10^{-7}$ after 6 iterations, which is where our strategy will stop the iterations (we continue them, to illustrate that $\lambda_{k}$ as well as the errors stay essentially unchanged).

\section{2 | Combined deconvolution and inpainting}

The second test problem is a 1D problem that combines deconvolution with inpainting. We first create an $n \times n$ Toeplitz matrix $A_{\text {full }}$ (with $n=216$ ) which is similar to the one from the test problem phillips from REGULARIZATION ToOLS, except that we change the point spread function to have half the width. Then we remove rows 71-126 of this matrix to obtain (using MATLAB notation) $A=A_{\text {full }}([1: 70,127: 216],:) \in \mathbb{R}^{160 \times 216}$. The exact solution $x_{\text {exact }}$ has elements $\sin (1.5 \pi i / n)+\cos (0.1 \pi i / n)$ and the exact right-hand side is $b_{\text {exact }}=A x_{\text {exact }}$ (think of $b_{\text {exact }}$ as the blurred version of $x_{\text {exact }}$ with the middle 56 elements removed, cf. Fig. 3). We then add Gaussian noise with relative noise level $\eta=10^{-4}$.

The numerical results are shown in Figure 3, using the augmentation subspace

$$
\mathcal{W}_{3}=\operatorname{span}\left\{w_{1}, w_{2}, w 3\right\}, \quad w_{1}=(1,1, \ldots, 1)^{T}, \quad w_{2}=(1,2, \ldots, n)^{T}, \quad w_{3}=\left(1,4, \ldots, n^{2}\right)^{T}
$$

The LSQR methods seeks to approximate a minimum-norm solution and therefore even the best solution has a large error in the middle where the method seeks to introduce small elements. The best HEB solutions are much better, because the augmentation subspace $W_{3}$ in (14) provides basis vectors that - together with the Tikhonov regularization - are able to "fill the gap" in the middle of the solution. The HEB method with a fixed $\lambda$ depends on a good a priori choice - otherwise many values have to be tried. The HEB method with $\lambda_{k}$ chosen by GCV works very well, and no prior knowledge about the noise level or the regularization parameter is required. 


\section{5 | CONCLUSION}

We demonstrated how to augment the bidiagonalization algorithm underlying LSQR such that it computes solutions in an enriched subspace consisting of the standard Krylov subspace plus a low-dimensional linear subspace that represents additional prior information about the solution. We also demonstrated how to add standard-form Tikhonov regularization, thus arriving at a hybrid enriched bidiagonalization algorithm that can choose the regularization parameter adaptively in each iteration. We discussed implementation issues and gave a few illustrative numerical examples.

\section{References}

1. Hansen PC. Discrete Inverse Problems: Insight and Algorithms. Philadelphia: SIAM; 2010.

2. van der Sluis A, van der Vorst HA. SIRT- and CG-type methods for the iterative solution of sparse linear least-squares problems. Lin. Alg. Appl. 1990;130:257-302.

3. Calvetti D, Lewis B, Reichel L. On the regularizing properties of the GMRES method. Numer. Mat. 2002;91:605-625.

4. Kilmer ME, Stewart GW. Iterative regularization and MINRES. SIAM J. Matrix Anal. Appl. 1999;21:613-628.

5. Calvetti D, Lewis B, Reichel L. GMRES-type methods for inconsistens systems. Lin. Alg. Appl. 2000;316:157-169.

6. Hanke M. Conjugate Gradient Type Methods for Ill-Posed Problems. Pitman Research Notes in Mathematics 327, Harlow: Longman; 1995.

7. Jensen TK, Hansen PC. Iterative regularization with minimum-residual methods. BIT. 2007;47:103-120.

8. Gazzola S, Novati P, Russo MR. On Krylov projection methods and Tikhonov regularization. Electron. Trans. Numer. Anal. 2015;44:83-123.

9. Baglama J, Reichel L. Augmented GMRES-type methods. Num. Lin. Alg. Appl. 2007;14:337-350.

10. Calvetti D, Reichel L, Shuibi A. Enriched Krylov subspace methods for ill-posed problems. Lin. Alg. Appl., 2003;362:257273.

11. Kuroiwa N, Nodera T. The adaptive augmented GMRES method for solving ill-posed problems. Mercer GN, Roberts AJ (Eds.), Proceedings of the 14th Biennial Computational Techniques and Applications Conference, CTAC-2008, ANZIAM J., 50 (2008), pp. C654-C667.

12. Dong $\mathrm{Y}$, Garde H, Hansen PC. $\mathrm{R}^{3}$ GMRES: including prior information in GMRES-type methods for discrete inverse problems. Electronic Transactions on Numerical Analysis, 2014;42:136-146.

13. Paige CC, Saunders MA. LSQR: an algorithm for sparse linear equations and sparse least squares. ACM Trans. Math. Soft. 1982;8:43-71.

14. Chung J, Nagy JG, O’Leary DP. A weighted-GCV method for Lanczos-hybrid regularization. Electronic Transactions on Numerical Analysis 2008;28:149-167.

15. Hansen PC, Jørgensen JS. AIR Tools II: algebraic iterative reconstruction methods, improved implementation. Numer. Algo, online, doi: 10.1007/s11075-017-0430-x.

16. Gazzola S, Hansen PC, Nagy JG. IR Tools: a MATLAB package of iterative regularization methods and large-scale test problems. Numer. Algo., to appear.

17. Golub GH, Van Loan CF. Matrix Comutations, 4th ed. Baltimore: Johns Hopkins University Press; 2013.

18. Hansen PC. Regularization Tools Version 4.0 for Matlab 7.3. Numer. Algo. 2007;46:189-194. 